\title{
On Bisectors for Different Distance Functions
}

\author{
Christian Icking* Rolf Klein* Lihong $\mathrm{Ma}^{*}$ \\ Stefan Nickel ${ }^{* *}$ Ansgar Weißler**
}

August 1998

\begin{abstract}
Let $\gamma_{C}$ and $\gamma_{D}$ be two convex distance functions in the plane with convex unit balls $C$ and $D$. Given two points, $p$ and $q$, we investigate the bisector, $B(p, q)$, of $p$ and $q$, where distance from $p$ is measured by $\gamma_{C}$ and distance from $q$ by $\gamma_{D}$. We provide the following results. $B(p, q)$ may consist of many connected components whose precise number can be derived from the intersection of the unit balls, $C$ and $D$. The bisector can contain bounded or unbounded 2-dimensional areas. Even more surprising, pieces of the bisector may appear inside the region of all points closer to $p$ than to $q$.

If $C$ and $D$ are convex polygons over $m$ and $n$ vertices, respectively, the bisector $B(p, q)$ can consist of at most $\min (m, n)$ connected components which contain at most $2(m+n)$ vertices altogether. The former bound is tight, the latter is tight up to an additive constant. We also present an optimal $O(m+n)$ time algorithm for computing the bisector.
\end{abstract}

Key words: Bisector, convex distance function, gauge, location problem, norm, Voronoi diagram.

*FernUniversität Hagen, Praktische Informatik VI, D-58084 Hagen, Germany.

** Universität Kaiserslautern, Fachbereich Mathematik, D-67653 Kaiserslautern, Germany. This research was supported by the DFG in the projects "Competitive Path Planning" and "Discrete Location Theory Problems" within the research cluster "Efficient Algorithms for Discrete Problems and their Applications". 


\section{Introduction}

The topic of this paper is a type of distance measure that is usually called a norm ${ }^{1}$ in mathematics, a convex distance function in computational geometry, and a gauge in location theory.

Let $C$ be a compact convex set in the plane containing the origin in its interior. Given a point $x$, we denote by $\gamma_{C}(x)$ the factor by which $C$ must be scaled to make its boundary pass through $x$. This number is used for measuring the distance of $x$ from the origin.

To measure the distance of $x$ from an arbitrary point $p$ we first translate $C$ such that its center lies at $p$, and then proceed as before. For a fixed site $p$ and a variable point $x$, let $d_{p}(x)$ denote the resulting distance measure. Clearly, the translate of $C$ is the unit ball of $d_{p}(x)$.

Similarly, let $D$ be another convex set like $C$, let $q$ be another point in the plane, and let $d_{q}(x)$ be the measure based on $D$ for the distance from site $q$ to point $x$. In this paper we are studying the bisector

$$
B(p, q):=\left\{x \in \mathbb{R}^{2}: d_{p}(x)=d_{q}(x)\right\}
$$

of $p$ and $q$ with respect to $d_{p}(x)$ and $d_{q}(x)$.

From a computational geometer's point of view, this can be seen as a first step to the study of the Voronoi diagram of $m \geq 2$ sites $p_{i}$, each of which is assigned its own distance measure, $d_{p_{i}}(x)$. It consists of Voronoi regions, one to each site $p_{i}$ which contains all points $x$ in the plane satisfying

$$
d_{p_{i}}(x)<d_{p_{j}}(x)
$$

for all $j \neq i$. Two special cases of this problem are well-known. First, if all unit balls of the measures $d_{p_{i}}(x)$ are translates of each other, we are faced with the Voronoi diagram of $m$ points with respect to one convex distance function. This problem has first been studied by Chew and Drysdale [3]. The bisector of two points is homeomorphic to a line [4, 7], the Voronoi regions

\footnotetext{
${ }^{1}$ If the measure is symmetric, which we do not require here.
} 
are star-shaped, and the Voronoi diagram is of complexity $O(m)$; it can be constructed within time $O(m \log m)$.

Second, if the unit balls are Euclidean circles centered at $p_{i}$, with possibly different radii, then the Voronoi diagram of points $p_{i}$ with multiplicative weights results. Aurenhammer and Edelsbrunner [1] have shown that the bisector of two differently weighted points equals a circle. The Voronoi diagram contains $O\left(n^{2}\right)$ many edges, faces, and vertices; it can be computed in $O\left(n^{2}\right)$ time. See Aurenhammer and Klein [2] for more information on both types of Voronoi diagrams.

There is a general approach to constructing such Voronoi diagrams due to Edelsbrunner and Seidel [5]. Let the $m$ unit balls expand over time, at the same speed each, resulting in an arrangement of $m$ three-dimensional cones whose apices are located at the point sites in the $(x, y)$-plane. Projecting its lower envelope onto the $(x, y)$-plane results in the Voronoi diagram. This approach works in higher dimensions, too, but it does not directly provide insight into the structure of the resulting diagrams. However, the structural properties of Voronoi diagrams based on convex distance functions are quite surprising; see [7], for example.

The bisector of two point sites with respect to different convex distance measures is also interesting to location theory; see e.g. Hamacher [6]. There are at least two different areas where bisectors are needed. In a planar location problem we are typically looking for locations of one or more new facilities with respect to a given set of clients. The location of the clients as well as the locations of the new facilities are represented by points.

If we locate several new facilities that provide a similar type of service, like supermarkets, drugstores, etc., not every client will go to all new facilities. A reasonable assumption is that a client is only visiting the new facility which is closest. Moreover, we would like to allow every client to have her own distance function, in order to reflect different travel conditions in different areas. Thus, for a given set of new locations the allocations of clients to new facilities is described by a Voronoi diagram, with the new facilities as 
sites to which different distance functions are assigned. For the case where all distance functions are identical the interested reader may find in [10] a survey on how to use Voronoi diagrams in location theory.

Recently (see $[9,11]$ and references therein) a new objective function for location problems has been proposed that includes, as special cases, all of the classical objective functions of location theory, i. e. median, center, and centdian. This new objective function is only pointwise defined and changes each time a potential facility $x$ crosses the bisector of two client points. Therefore, the complexity of solving a so-called Ordered Weber Problem relies heavily on the computation of bisectors.

This paper is organized as follows. In Section 2 we state the basic definitions. Then, in Section 3, we describe structural properties of the bisector of two points. It turns out that $B(p, q)$ can break up into many disconnected pieces; this cannot occur if the unit balls, $C$ and $D$, are the same. In the general case, we show how to determine the connected components, both in type and in number, from the intersection of $C$ and $D$.

It has been known from the case $C=D$ that the bisector $B(p, q)$ may contain unbounded two-dimensional pieces; this happens when the line through $p$ and $q$ is parallel to an edge in the unit ball's boundary. We show that the bisector based on two different unit balls can contain bounded two-dimensional pieces as well.

Even more surprising - and somewhat disturbing - is the following phenomenon. Inside the region of all points $x$ closer to $p$ than to $q$ there may be a piece of the bisector, $B(p, q)$. In other words, there can be "weak" Voronoi edges with the same Voronoi region on either side!

In Section 4 we study the case where the unit balls are convex polygons of $m$ and $n$ vertices, respectively. We show that the bisector can consist of at $\operatorname{most} \min (m, n)$ many connected components, and that this bound is tight. Altogether, the bisector can consist of at most $2(m+n)$ many linear pieces, and this bound is tight up to an additive constant. Finally, we present an algorithm that constructs the bisector within optimal time $O(m+n)$. 


\section{Definitions}

Let $C \subseteq \mathbb{R}^{2}$ be a compact convex set containing the origin $O$ in its interior. The gauge $\gamma_{C}$ defined as

$$
\gamma_{C}: \mathbb{R}^{2} \rightarrow \mathbb{R}, \quad x \mapsto \inf \{\lambda>0: x \in \lambda C\}
$$

fulfills the properties of a, not necessarily symmetric, distance function:

$$
\begin{array}{rll}
\gamma_{C}(x) \geq 0 & \forall x \in \mathbb{R}^{n} & \text { non-negativity } \\
\gamma_{C}(x)=0 \quad \Leftrightarrow \quad x=0 & \text { definiteness } \\
\gamma_{C}(\lambda x)=\lambda \gamma_{C}(x) \quad \forall x \in \mathbb{R}^{n}, \forall \lambda \geq 0 & \text { positive homogeneity } \\
\gamma_{C}(x+y) \leq \gamma_{C}(x)+\gamma_{C}(y) \quad \forall x, y \in \mathbb{R}^{n} & \text { triangular inequality }
\end{array}
$$

Obviously $C$ is the unit ball of the distance function $\gamma_{C}$. It is clear from the definition that $\gamma_{C}(y)=1$ iff $y$ lies on the boundary of $C$ and that $\gamma_{C}(x)$ is the factor by which we have to scale $C$ to make $x$ lie on its boundary, see Figure 1.

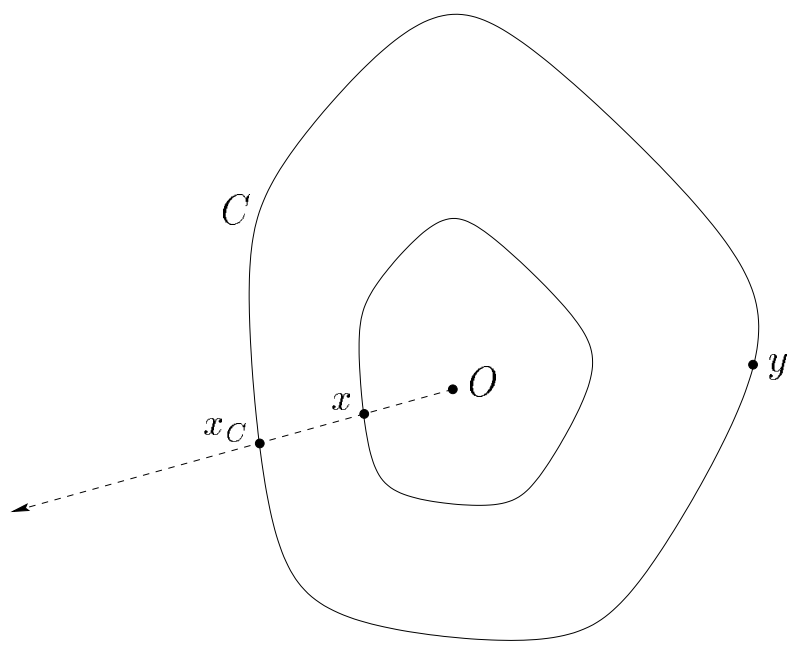

Figure 1: Convex compact set $C$ defining a gauge $\gamma_{C}$.

The analytic definition (1) of $\gamma_{C}$ is equivalent to the geometric definition

$$
\gamma_{C}: \mathbb{R}^{2} \rightarrow \mathbb{R}, \quad x \mapsto \frac{l_{2}(x)}{l_{2}\left(x_{C}\right)}
$$


where $l_{2}$ is the Euclidean norm and $x_{C}$ is the uniquely determined intersection point of the boundary of $C$ and the ray $\overrightarrow{O x}$ from $O$ through $x$.

The distance from a point $x$ to a point $y$ with respect to $\gamma_{C}$ is defined as $\gamma_{C}(y-x)$. Notice that in general $\gamma_{C}(y-x) \neq \gamma_{C}(x-y)$, due to non-symmetry.

In this paper, we consider a situation with two sites $p, q \in \mathbb{R}^{2}$ and two unit balls $C$ and $D$. The distance from site $p$ to a point $x$ is always measured with respect to $\gamma_{C}$. We use $\gamma_{p}$ as a synonym for $\gamma_{C}$, and let $d_{p}(x):=\gamma_{p}(x-p)$. Analogue notations $\gamma_{q}=\gamma_{D}$ and $d_{q}$ are used for site $q$ and unit ball $D$. The bisector of $p$ and $q$ with respect to $\gamma_{p}$ and $\gamma_{q}$ is defined by

$$
B(p, q):=\left\{x \in \mathbb{R}^{2}: d_{p}(x)=d_{q}(x)\right\}
$$

The set of all points in the plane which can be reached faster from $p$ with respect to $\gamma_{p}$ than from $q$ with respect to $\gamma_{q}$ is denoted by

$$
R(p, q):=\left\{x \in \mathbb{R}^{2}: d_{p}(x)<d_{q}(x)\right\}
$$

\section{Structural properties}

We choose a coordinate system such that $p$ and $q$ lie on a horizontal line and the abscissa of $p$ is smaller than the one of $q$.

If we translate a geometric object by a vector $v$, we denote the new object by $A^{v}$, e.g. $C^{p}$ denotes the unit ball $C$ centered at site $p$.

For $x \neq p$ let the foot-point, $x_{p}$, of $x$ with respect to $p$ be the unique intersection point of the ray $\overrightarrow{p x}$ and the boundary of $C^{p}$, and analogously for $x_{q}$.

Lemma 1 For a point $x \in B(p, q)$ of the bisector, the line $x_{p} x_{q}$ through the two foot-points is parallel to the line $p q$. Conversely, if there are two boundary points on a horizontal line, point $u$ on $C^{p}$ and point $v$ on $D^{q}$, such that the rays $\overrightarrow{p u}$ and $\overrightarrow{q v}$ intersect, then this intersection is a bisector point. 
Proof. If $x$ lies on the line $p q$ then $x_{p}$ and $x_{q}$ also do.

Otherwise we consider the triangles $\triangle(x, p, q)$ and $\triangle\left(x, x_{p}, x_{q}\right)$. From $(2)$ we know that

$$
\frac{l_{2}(x-p)}{l_{2}\left(x_{p}-p\right)}=\frac{l_{2}(x-q)}{l_{2}\left(x_{q}-q\right)} .
$$

This implies that the two triangles are homothetic, i. e. their sides are parallel.

The converse is a direct consequence of (2) and (3).

From this simple property we can already conclude some interesting facts about the bisector. Consider a point $u$ on the boundary of $C^{p}$. Which bisector points do have the foot-point $u$ with respect to $p$ ? Of course, these are points on the ray $\overrightarrow{p u}$.

From Lemma 1 we know that their foot-points with respect to $q$ must lie on the line through $u$ parallel to $p q$ and on the boundary of $D^{q}$. This intersection consists of zero, one, two points or a line segment, and these are the only possible "partner foot-points".

Corollary 2 The ray $\overrightarrow{p u}$ may only contain zero, one, or two bisector points, or, in the special case of a line segment of possible partner foot-points for $u$, a line segment or a ray.

See Figure 2 for examples of all cases.

Now let us compare two gauges in a certain direction. We say that gauge $\gamma_{p}$ is stronger than gauge $\gamma_{q}$ in direction $e \in \mathbb{R}^{2}$, if $\gamma_{p}(e)<\gamma_{q}(e)$. Note that " $<$ " means stronger since the wider the unit ball extends in a direction the smaller factor is necessary to scale the ball until it reaches a certain point.

Lemma 3 Assume that gauge $\gamma_{q}$ is stronger than $\gamma_{p}$ in a direction e. Then the ray from $p$ in direction e contains exactly one point of the bisector $B(p, q)$.

Proof. We define the function $f: \mathbb{R}^{+} \rightarrow \mathbb{R}$ with

$$
f(\lambda):=d_{q}(p+\lambda e)-d_{p}(p+\lambda e) .
$$

A point $p+\lambda e$ is on the bisector iff $f(\lambda)=0$. 

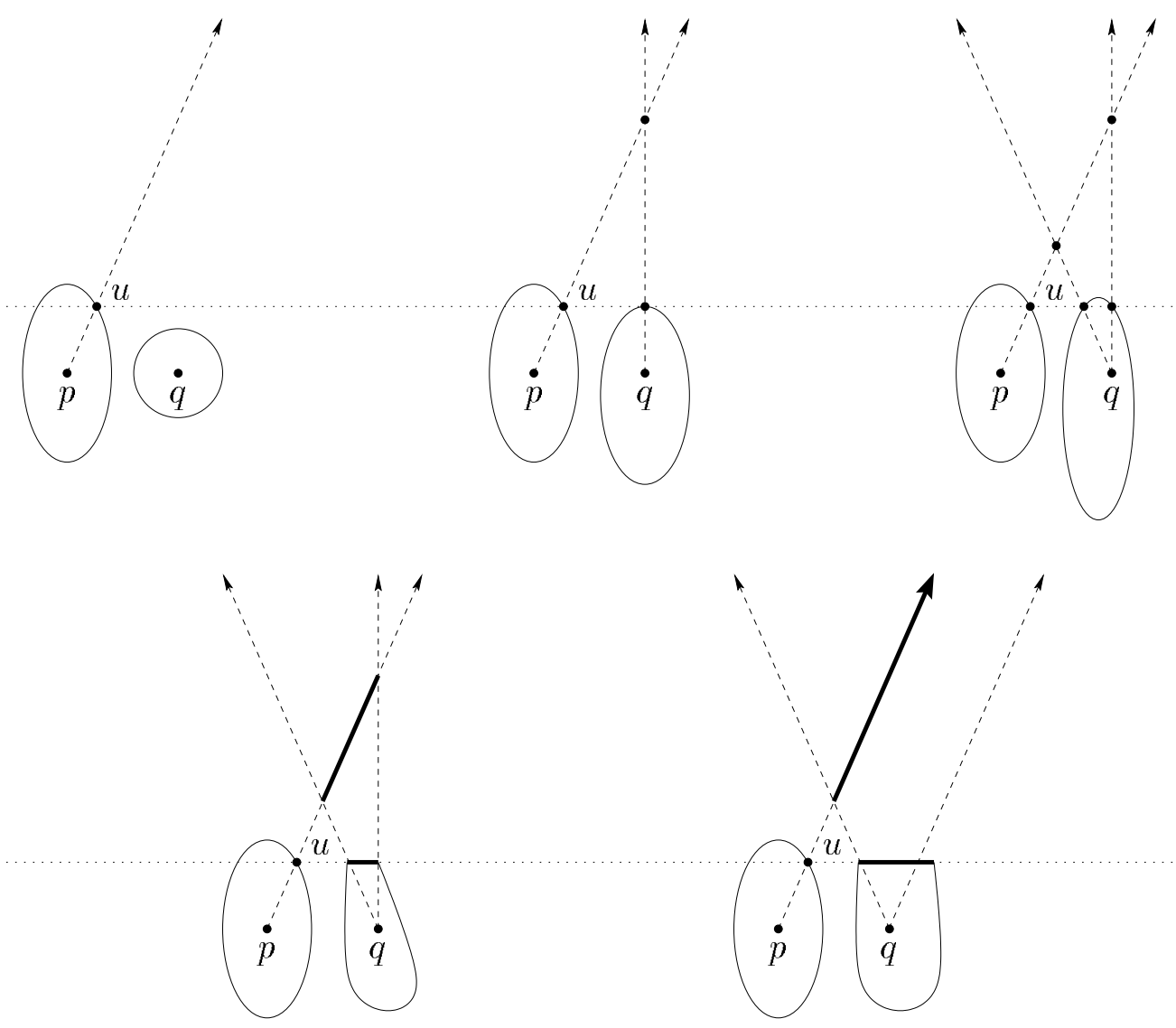

Figure 2: The ray $\overrightarrow{p u}$ may contain zero, one, or two bisector points, or, in a special case, a bisector segment or ray.

We show that $f$ is a convex function. For $\mu \in[0,1]$ and $\lambda_{1}, \lambda_{2} \in[0, \infty)$ we have

$$
\begin{aligned}
f(\mu & \left.\lambda_{1}+(1-\mu) \lambda_{2}\right) \\
& =\gamma_{q}\left(p+\left(\mu \lambda_{1}+(1-\mu) \lambda_{2}\right) e-q\right)-\gamma_{p}\left(\left(\mu \lambda_{1}+(1-\mu) \lambda_{2}\right) e\right) \\
& =\gamma_{q}\left(\mu\left(p+\lambda_{1} e-q\right)+(1-\mu)\left(p+\lambda_{2} e-q\right)\right)-\gamma_{p}\left(\mu \lambda_{1} e+(1-\mu) \lambda_{2} e\right) \\
& \leq \mu \gamma_{q}\left(p+\lambda_{1} e-q\right)+(1-\mu) \gamma_{q}\left(p+\lambda_{2} e-q\right)-\mu \gamma_{p}\left(\lambda_{1} e\right)+(1-\mu) \gamma_{p}\left(\lambda_{2} e\right) \\
& =\mu f\left(\lambda_{1}\right)+(1-\mu) f\left(\lambda_{2}\right) .
\end{aligned}
$$


Furthermore, $f(0)=d_{q}(p)$ is positive and, again using the triangle inequality,

$$
f(\lambda)=\gamma_{q}(p+\lambda e-q)-\gamma_{p}(\lambda e) \leq \gamma_{q}(p-q)+\lambda\left(\gamma_{q}(e)-\gamma_{p}(e)\right)
$$

and therefore $\lim _{\lambda \rightarrow \infty} f(\lambda)=-\infty$ because $\gamma_{q}(e)-\gamma_{p}(e)$ is negative.

A convex function which tends to $-\infty$ for $\lambda \rightarrow \infty$ must be strictly decreasing. As one can see from (6), the only zero of $f$ must be contained in $\left(0, \frac{\gamma_{q}(p-q)}{\gamma_{p}(e)-\gamma_{q}(e)}\right]$.

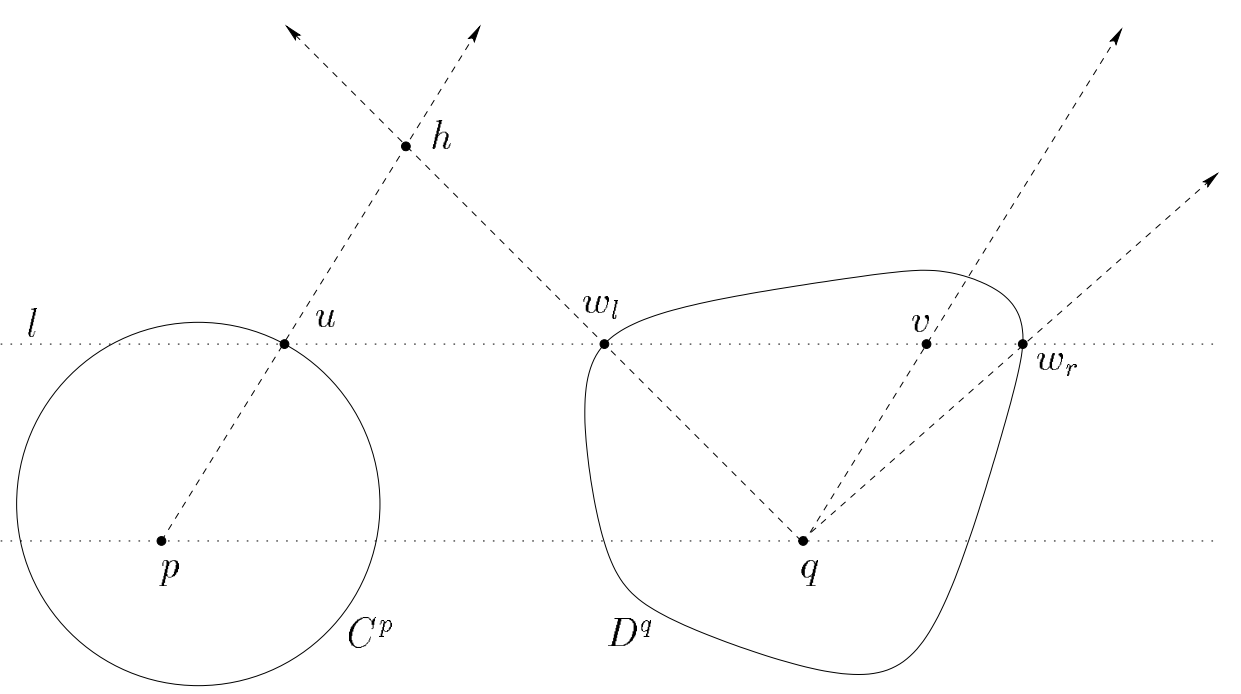

Figure 3: Point $h$ is on the bisector, and ray $\overrightarrow{p h}$ does not hit any other bisector point because $\overrightarrow{p h}$ is parallel to $\overrightarrow{q v}$ and $\gamma_{q}$ is stronger than $\gamma_{p}$ in this direction.

For a geometrical explanation see Figure 3 . Let $u$ be the point on the boundary of $C^{p}$ such that ray $\overrightarrow{p u}$ has direction $e$, let $l$ be the horizontal line through $u$, and let $v$ be the intersection of $l$ and the ray from $q$ in direction $e$.

Since $\gamma_{q}$ is stronger than $\gamma_{p}$ in direction $e$, point $v$ lies in the interior of $D^{q}$. Therefore, $l \cap \partial D^{q}$, contains exactly two points, $w_{l}$ to the left and $w_{r}$ to the right, which are the only possible partner foot-points for $u$. By comparing the slopes, we note that the ray $\overrightarrow{q w_{l}}$ must intersect $\overrightarrow{p u}$ while $\overrightarrow{q w_{r}}$ can not. Therefore, we have exactly one bisector point. 
As we have already seen, it is important how the boundaries of the two unit balls intersect horizontal lines. Normally, for one ball we have one intersection point facing the other and another intersection point on the back. Therefore we divide the boundary of a unit ball into two disjoint parts.

The top point of $C$, denoted by $t_{p}$, is the leftmost point of the intersection of $C$ and the horizontal tangent to $C$ from above, and the bottom point of $C, b_{p}$, is the corresponding point from below. The top and bottom points of $D$ are defined analogously, i. e. "leftmost" is replaced by "rightmost". See Figure 4, where the balls and the points are shifted by $p$ resp. $q$.

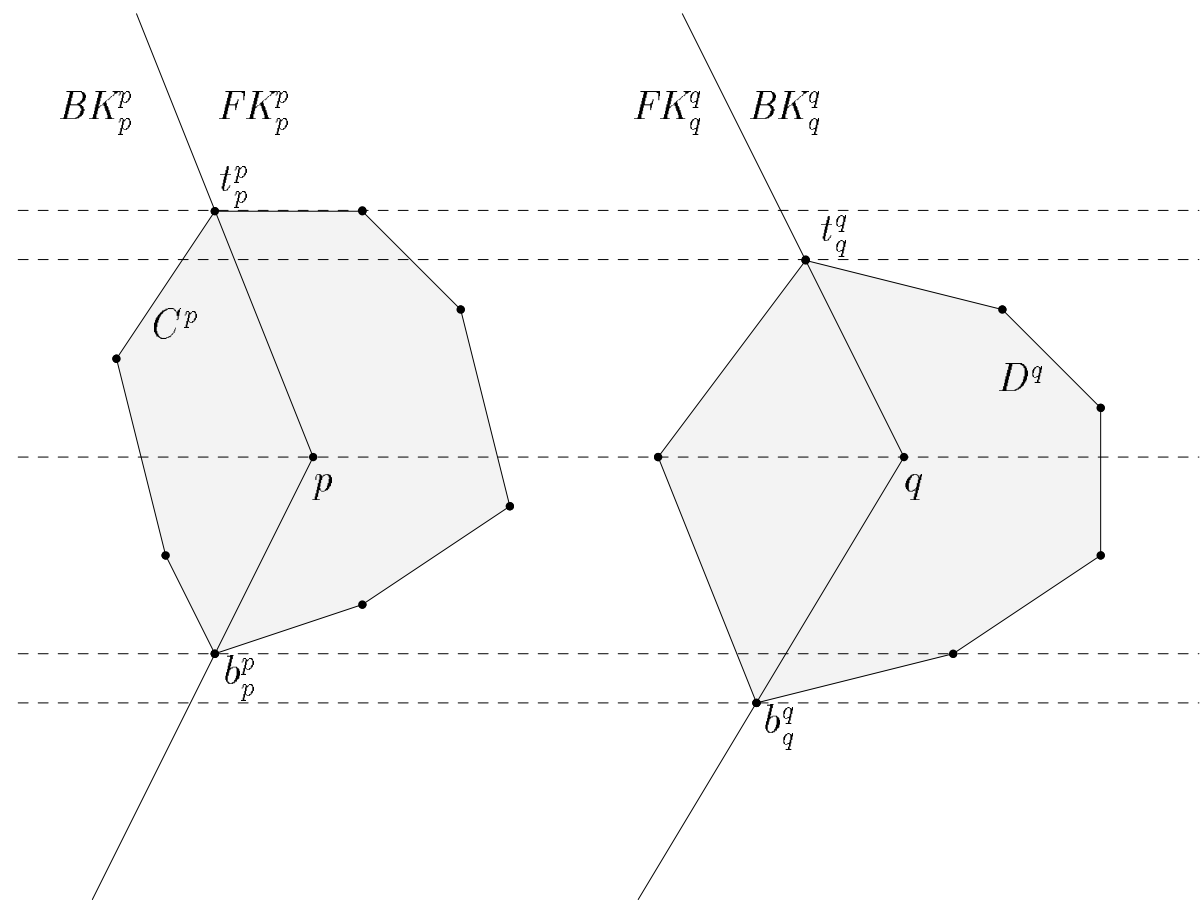

Figure 4: Definition of top and bottom points, face and back cones.

For each unit ball, the plane is divided into two cones separated by two rays. The face cone of $C, F K_{p}$, is the closed cone containing $q-p$ with boundary $\overrightarrow{O t_{p}}$ and $\overrightarrow{O b_{p}}$, and the back cone of $C, B K_{p}$, is its open complement. The face and back cones of $D$ are defined analogously. In Figure 4 the shifted 
cones $F K_{p}^{p}$ etc. are shown.

The bisector $B(p, q)$ is decomposed into four parts, depending on the intersection of these cones.

$$
\begin{aligned}
& F F-B(p, q):=B(p, q) \cap F K_{p}^{p} \cap F K_{q}^{q} \\
& F B-B(p, q):=B(p, q) \cap F K_{p}^{p} \cap B K_{q}^{q} \\
& B F-B(p, q):=B(p, q) \cap B K_{p}^{p} \cap F K_{q}^{q} \\
& B B-B(p, q):=B(p, q) \cap B K_{p}^{p} \cap B K_{q}^{q}
\end{aligned}
$$

The "face to face" bisector $F F-B(p, q)$ is never empty, while there are equivalent criteria for the emptiness of the other parts, as the next lemma shows.

\section{Lemma 4}

(i) $F F-B(p, q) \neq \emptyset$.

(ii) $B F-B(p, q) \neq \emptyset$ iff a direction $e \in B K_{p} \cap F K_{q}$ exists such that $\gamma_{q}$ is stronger than $\gamma_{p}$ in this direction.

(iii) $F B-B(p, q) \neq \emptyset$ iff a direction $e \in F K_{p} \cap B K_{q}$ exists such that $\gamma_{p}$ is stronger than $\gamma_{q}$ in this direction.

(iv) $B B-B(p, q) \neq \emptyset$ iff $\left(\partial C \cap B K_{p}\right) \cap\left(\partial D \cap B K_{q}\right) \neq \emptyset$.

\section{Proof.}

(i) We show that the line segment $\overline{p q}$, which lies in $F K_{p}^{p} \cap F K_{q}^{q}$, contains a bisector point.

The function $f:[0,1] \rightarrow \mathbb{R}$ defined by

$$
\begin{aligned}
f(\lambda) & :=d_{p}(\lambda p+(1-\lambda) q)-d_{q}(\lambda p+(1-\lambda) q) \\
& =\gamma_{p}((1-\lambda)(q-p))-\gamma_{q}(\lambda(p-q)) \\
& =(1-\lambda) d_{p}(q)-\lambda d_{q}(p)
\end{aligned}
$$

has a zero at $\lambda_{0}:=\frac{d_{p}(q)}{d_{p}(q)+d_{q}(p)} \in(0,1)$, which means that $x_{0}:=\lambda_{0} p+$ $\left(1-\lambda_{0}\right) q \in \overline{p q}$ is on the bisector. 
(ii) If $\gamma_{q}$ is stronger than $\gamma_{p}$ in direction $e \in B K_{p} \cap F K_{q}$ then we know from Lemma 3 that there is a point $x=p+\mu e \in B(p, q)$ on the ray from $p$ in this direction. This ray is contained in $\left(B K_{p} \cap F K_{q}\right)^{p}=B K_{p}^{p} \cap F K_{q}^{p} \subset B K_{p}^{p} \cap F K_{q}^{q}$ because $F K_{q}^{p}$ is simply $F K_{q}^{q}$ translated to the left, thus $x \in B F-B(p, q)$.

Conversely, assume that $x \in B F-B(p, q)$, i. e. $x \in B K_{p}^{p} \cap F K_{q}^{q}$. We choose $e:=x-q$, and we have $x-q \in\left(B K_{p}^{p} \cap F K_{q}^{q}\right)^{-q}=B K_{p}^{p-q} \cap F K_{q}$. But $B K_{p}^{p-q}$ is just $B K_{p}$ translated to the left and therefore a subset of it, thus $e \in B K_{p} \cap F K_{q}$.

If the point $x$ is on the line $p q$ then, by using (4), it is easy to obtain that $\gamma_{q}$ is stronger than $\gamma_{p}$ in this direction. Otherwise, we consider the footpoint, $x_{p}$, of $x$. Since it lies in the back cone $B K_{p}^{p}, x_{p}$ is the leftmost point in the intersection of $C^{p}$ and the horizontal line, $l$, through $x_{p}$. The ray from $p$ in direction $e$ is parallel to $\overrightarrow{q x}$ and intersects the line $l$ to the left of $x_{p}$, i. e. outside of $C^{p}$. But this implies that $\gamma_{q}$ is stronger than $\gamma_{p}$ in this direction.

(iii) This claim is obtained by interchanging $p$ and $q$ in (ii).

(iv) Let $x \in B B-B(p, q)$, and let $l$ be the horizontal line through the foot-points, as usual. Since the foot-points lie in the back cones, $x_{p}$ is the leftmost intersection point of $C^{p}$ and $l$, and $x_{q}$ is the rightmost intersection point of $D^{q}$ and $l$.

Now we consider the translated $D^{p}$, see Figure 5. The point $x_{q}+p-q$ on the boundary of $D^{p}$ lies to the left of $x_{p}$ on line $l$. From $x_{q}+p-q$, the boundary of $D^{p}$ runs to a point to the right of $p$ on line $p q$, while the boundary of $C^{p}$ runs from $x_{p}$ to a point to the left of $p$, therefore they must intersect, see point $y$ in Figure 5 .

To prove the converse, assume that $y \in\left(\left(\partial C \cap B K_{p}\right) \cap\left(\partial D \cap B K_{q}\right)\right)^{p}$, and w.l.o.g. $y$ lies above the line $p q$, see Figure 5 .

Due to convexity the upper part of $\partial C^{p} \cap B K_{p}^{p}$ from $y$ to $t_{p}^{p}$ lies to the right of $\partial D^{p} \cap B K_{q}^{p}$ from $y$ to $t_{q}^{p}$. A horizontal line anywhere in between $y$ and the lower of $t_{p}^{p}$ and $t_{q}^{p}$ intersects $\partial C^{p} \cap B K_{p}^{p}$ in $x_{p}$ and $\partial D^{q} \cap B K_{q}^{q}$ in $x_{q}$. It is clear that $\overrightarrow{p x_{p}}$ intersects $\overrightarrow{q x_{q}}$ in a point $x \in B B-B(p, q)$, because $x_{q}+p-q$ lies to the left of $x_{p}$. 


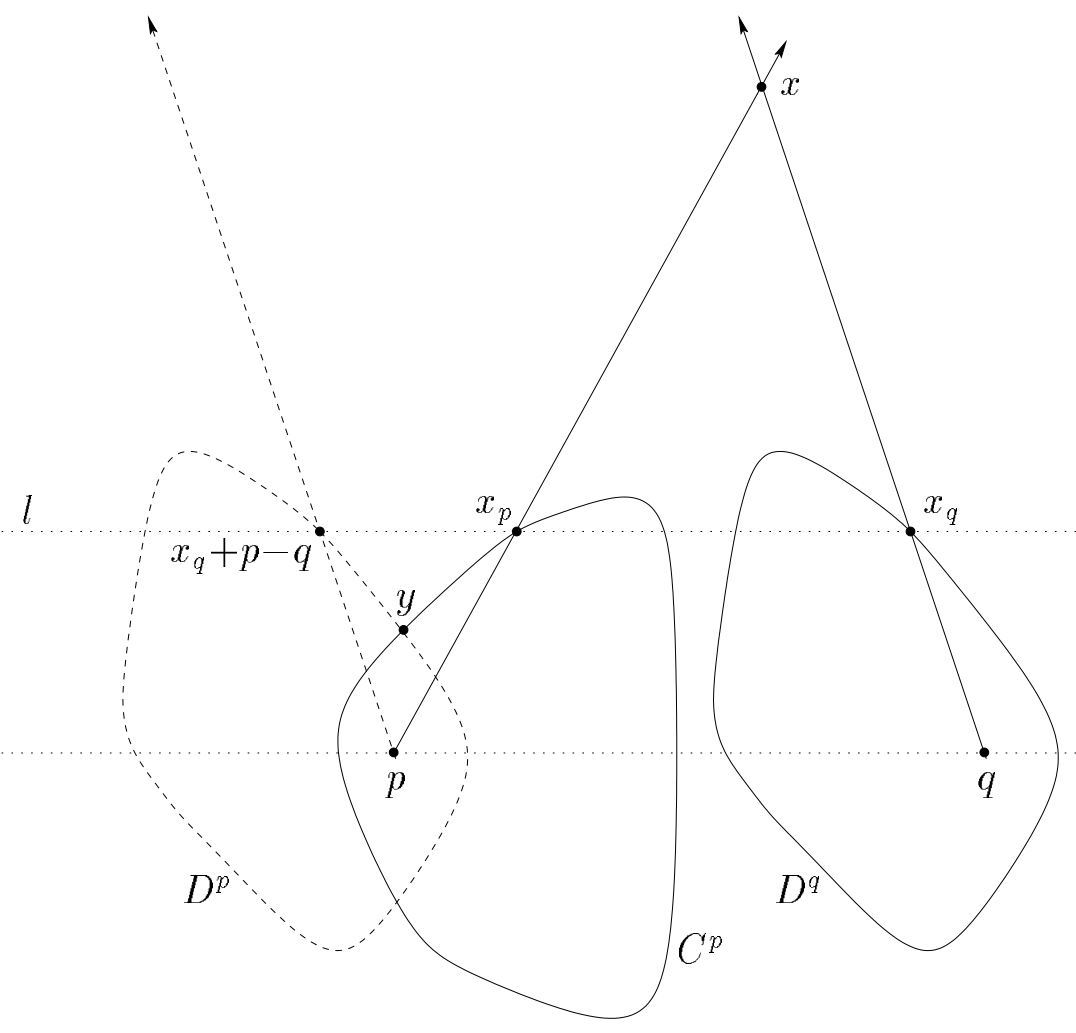

Figure 5: A bisector point, $x$, in the intersection of the two back cones.

Corollary 5 For two identical gauges $\gamma_{p}=\gamma_{q}$ we have $B(p, q)=F F-B(p, q)$. In other words the other potential bisector parts $F B-B(p, q), B F-B(p, q)$, and $B B-B(p, q)$ are empty.

Proof. The two gauges are equally strong in any direction, therefore $F B-B(p, q)$ and $B F-B(p, q)$ are empty due to Lemma 4 (ii) and (iii). Furthermore, we have $B K_{p} \cap B K_{q}=\emptyset$ by the definition, so $B B-B(p, q)$ is empty by Lemma 4 (iv).

Example 1 In the ordinary case of the Euclidean metric we have $B(p, q)=$ $F F-B(p, q)$ which is the perpendicular bisector of the line segment $\overline{p q}$. 
Lemma 6 If there is a bisector point $x$ on the ray from $p$ in direction $e \in F K_{q}$ then $\gamma_{q}(e) \leq \gamma_{p}(e)$. In case of $\gamma_{q}(e)=\gamma_{p}(e)$ the boundary of $D^{q}$ contains a line segment parallel to the line $p q$, and the foot point $x_{p}$ lies on the supporting line of this segment.

Proof. Let $x \in B(p, q)$ be a bisector point on the ray from $p$ in direction $e$ with foot-points $x_{p}$ and $x_{q}$. We know that the line $x_{p} x_{q}$ is parallel to $p q$. Let $u$ be the intersection point of the ray from $q$ in direction $e$ and the boundary of $D^{q}$. So $x_{q}$ must lie to the left of the ray $\overrightarrow{q u}$, therefore $\gamma_{p}(e) \geq \gamma_{q}(e)$ because of $e \in F K_{q}$. In case of $\gamma_{p}(e)=\gamma_{q}(e)$ the line $x_{p} u$ is parallel to $p q$, in other words $x_{p}, x_{q}$ and $u$ are collinear on a horizontal line. The two points $x_{q}$ and $u$ are in $F K_{q}^{q}$ and on the boundary of $D^{q}$, therefore the line segment $\overline{x_{q} u}$ is contained in $\partial D^{q}$, and the ray from $x$ in direction $e$ is in $B(p, q)$.

The next lemma shows that the number of connected components of the bisector $B(p, q)$ essentially depends on the number of intersections of the boundaries of the two unit balls.

\section{Lemma 7}

(i) $F F-B(p, q)$ is connected. It is separated from the rest of $B(p, q)$ iff $\partial C \cap \partial D \cap F K_{p} \cap F K_{q}$ is a set of exactly two points.

(ii) The number of the connected components in $B F-B(p, q)$ is equal to the number of the connected components of the set

$$
E:=\left\{e: e \in \partial C \cap B K_{p} \cap F K_{q} \text { and } \gamma_{q}(e)<\gamma_{p}(e)\right\}
$$

and analogously for $F B-B(p, q)$.

(iii) $B B-B(p, q)$ consists of at most two connected components, but they are connected to other parts of the bisector.

Proof. For the proof of (ii), we define a function $f: E \rightarrow B F-B(p, q)$ by $f(e)=x$, where $x$ is the unique bisector point on the ray from $p$ in direction $e$, according to Lemma 3 . 
We show that $f$ is continous. Let $e \in E$ and $x=f(e)$, let $V$ be a neighbourhood of $x$, and assume first that $e$ is not horizontal. Because $\gamma_{q}$ is stronger than $\gamma_{p}$ in direction $e$, there is a neighbourhood of $e$ in $E$ where this also holds, due to the continuity of $\gamma_{p}$ and $\gamma_{q}$. Let $e_{1}$ and $e_{2}$ be two points in this neighbourhood of $e$ which lie on opposite sides of $e$. We consider the two cones bounded by the rays $\overrightarrow{p f\left(e_{1}\right)}$ and $\overrightarrow{p f\left(e_{2}\right)}$ resp. $\overrightarrow{q f\left(e_{1}\right)}$ and $\overrightarrow{q f\left(e_{2}\right)}$. If only the neighbourhood of $e$ is chosen small enough the two cones intersect in a quadrilateral which is contained in $V$, thus $f$ is continous at $e$. For horizontal $e, f$ is also continous at this point by extension.

Due to continuity, the image $f\left(E_{1}\right) \subset B F-B(p, q)$ of a connected component $E_{1}$ of $E$ is also connected [8, Sect. 1.5, Th. 5]. Two connected components $E_{1}$ and $E_{2}$ of $E$ are separated by a direction $e \in \partial C \cap B K_{p} \cap F K_{q}$ with $\gamma_{q}(e) \geq \gamma_{p}(e)$. From $p$ in direction $e$ there is no bisector point, following Lemma 6 . Therefore, the images $f\left(E_{1}\right)$ and $f\left(E_{2}\right)$ are separated from each other by the ray from $p$ in direction $e$.

It remains to consider if there are bisector points $x \in B F-B(p, q)$ with $x \notin f(E)$. By Lemma 6 , for such direction $e$ from $p$ to $x$ we have $\gamma_{q}(e)=$ $\gamma_{p}(e)$, the foot-point $x_{p}$ lies on a horizontal line supporting a line segment on the boundary of $D^{q}$, and there are at most two such directions, namely the topmost and bottommost in the closure of $E$.

In such a case, the bisector contains a ray $R$ in this direction, see the last case in Figure 2. But there is a neighbornhood of $e$ in $E$ where $\gamma_{q}$ is stronger than $\gamma_{p}$, so by continuity $R$ is connected to $f\left(E_{1}\right)$ where $E_{1}$ is the connected component of $E$ with $e$ on its boundary.

The proofs of (i) and (iii) use very similar arguments. Remark that the boundaries of the two unit balls intersect at most two times in $\left(F K_{p} \cap F K_{q}\right) \cup$ $\left(B K_{p} \cap B K_{q}\right)$, due to convexity, once above the line $p q$ and once below. The upper one, if is lies in $F K_{p} \cap F K_{q}$, restricts the positions of the foot-points of $F F-B(p, q)$ from above, the lower one from below, and (i) follows.

There may be two possible parts of $B B-B(p, q)$ because $B K_{p}^{p} \cap B K_{q}^{q}$ may consist of two regions. One component of $B B-B(p, q)$, if it exists, always ends 
in a point whose foot-point is a top or bottom point, and therefore belongs to a face cone, which proves (iii).

Corollary 8 Let $k$ and $l$ be the number of connected components of $B F-B(p, q)$ resp. $F B-B(p, q)$ from Lemma $7(i i)$. The number of connected components of the whole bisector $B(p, q)$ is at least $\max \{1, k+l-3\}$ and at most $k+l+1$.

Proof. Except for the topmost and bottommost components of $B F-B(p, q)$ and $F B-B(p, q)$, which may be connected to $F F-B(p, q)$, the other components of $B F-B(p, q)$ and $F B-B(p, q)$ can not be connected within $B(p, q)$, and the lower bound follows. The upper bound is a direct consequence of Lemma 7 .

Corollary 9 If $\gamma_{q}$ is stronger than $\gamma_{p}$ in all directions then the bisector is a closed curve around $p$.

Proof. Similar to the proof of Lemma 7, we consider the function $f$ : $\partial C \rightarrow B(p, q)$ where $f(e)$ is the unique bisector point on the ray from $p$ in direction $e$, according to Lemma 3. Function $f$ is bijective and, as we have seen, also continous. The inverse of $f$ is also continous, since $\partial C$ is compact [8, Sect. 1.5, Th. 8], therefore $B(p, q)$ is homeomorphic to $\partial C$.

In the following we see examples concerning the four parts of the bisector, its number of components, and some special cases.

Example 2 In the case of two multiplicatively weighted Euclidean metrics with weights $0<w_{p}<w_{q}$ we have $B(p, q):=\left\{x \in \mathbb{R}^{2}: l_{2}(x-p) / w_{p}=\right.$ $\left.l_{2}(x-q) / w_{q}\right\}$ which is a circle containing $p$ in its interior; see [1] and Figure 6 .

Example 3 For odd $n$ we consider two regular $n$-gons which are rotated against each other. The set $E$ of Lemma 7 consists of $\frac{n-1}{2}$ connected components, therefore each of $B F-B(p, q)$ and $F B-B(p, q)$ has exactly that many pieces. Together with the one piece of $F F-B(p, q)$ which separates $p$ and $q$, the bisector consists of $n$ disconnected pieces. Figure 7 shows the bisector of two regular 7-gons. 


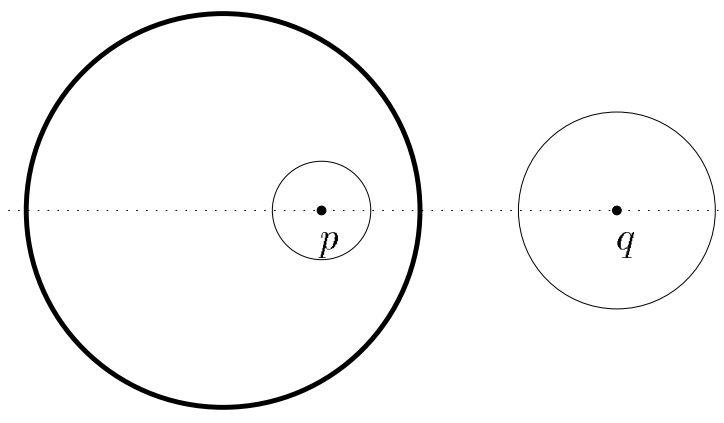

Figure 6: The bisector of two weighted Euclidean metrics is a circle.

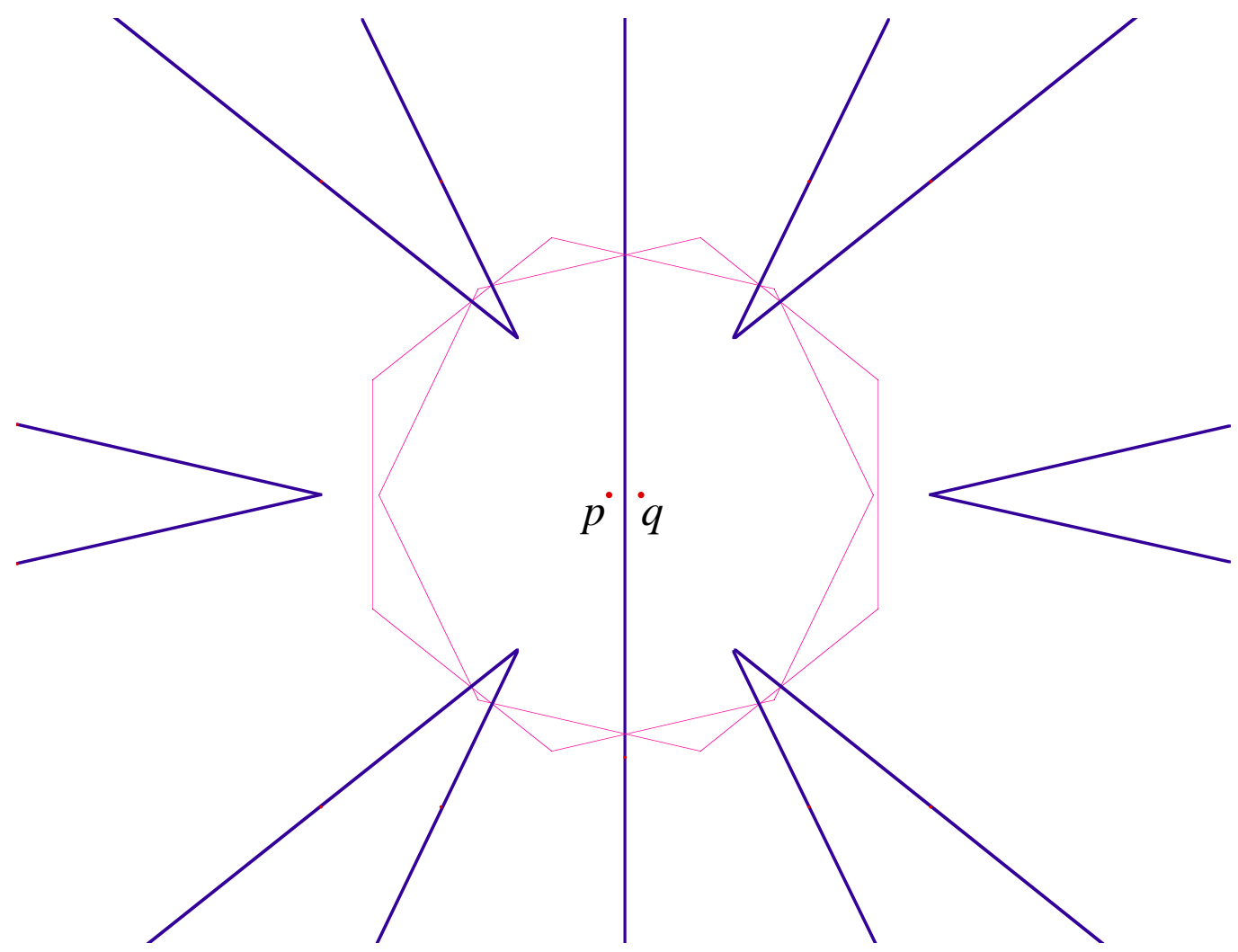

Figure 7: For two rotated 7-gons centered at $p$ resp. $q$, the bisector consists of 7 disonnected pieces. 
Example 4 There can be pieces of the bisector which do not separate the regions $R(p, q)$ and $R(q, p)$, as Figure 8 shows. Only $F F-B(p, q)$ may contain such non-separating, "weak" pieces. Here, as in Example $3, B B-B(p, q)$ is empty.

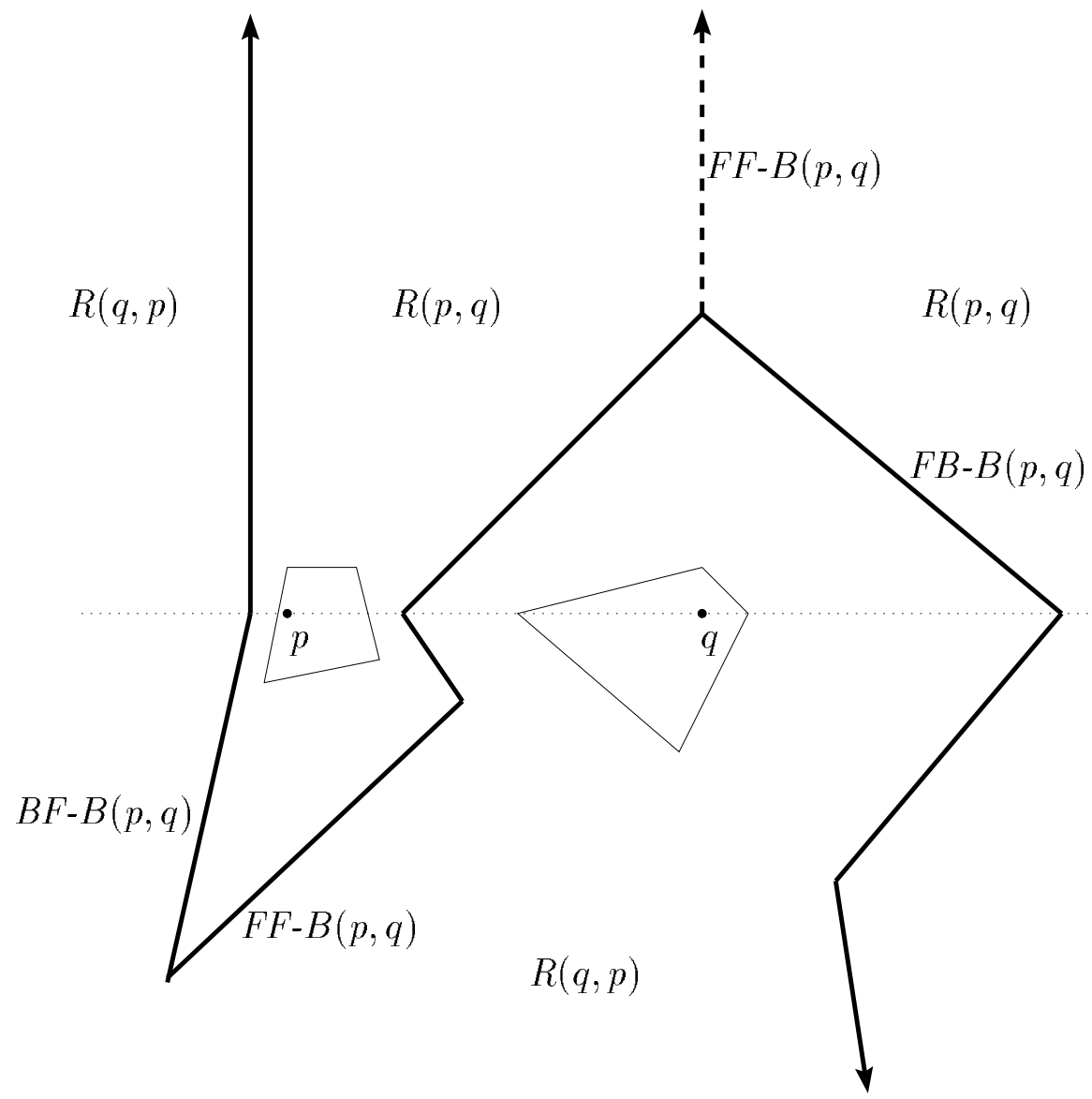

Figure 8: The piece of $B(p, q)$ drawn as a dashed line does not separate the two regions $R(p, q)$ and $R(q, p)$. 
Example 5 The bisector, more precisely $F F-B(p, q)$, can contain a bounded or unbounded area, if the boundaries of the two unit circles contain collinear horizontal line segments, see Figure 9.

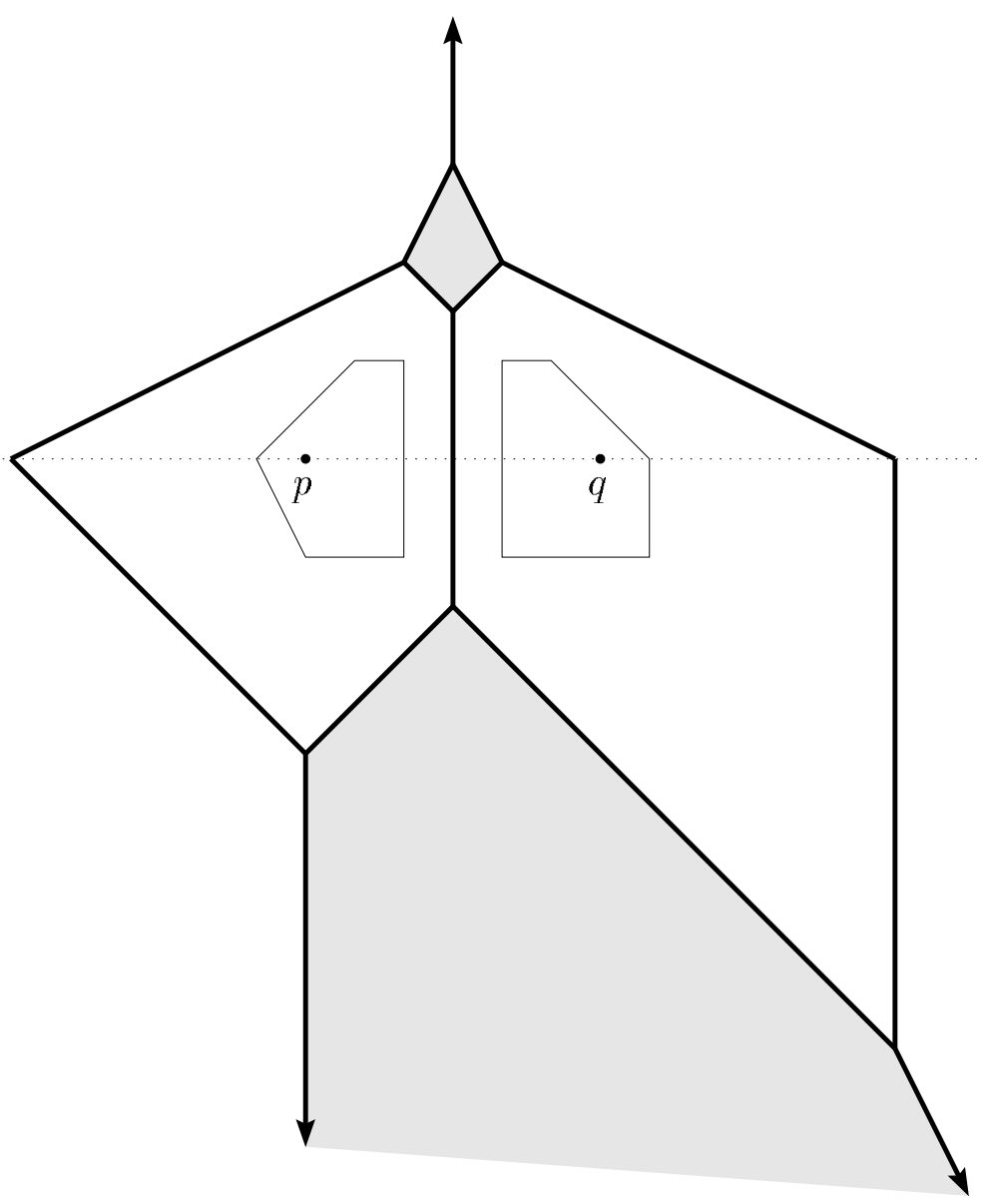

Figure 9: Bounded and unbounded areas in the bisector. 
Example 6 In Figure 10 we see a bisector for which all four parts, $F F-B(p, q)$, $B F-B(p, q), F B-B(p, q)$, and $B B-B(p, q)$, are not empty, and are connected.

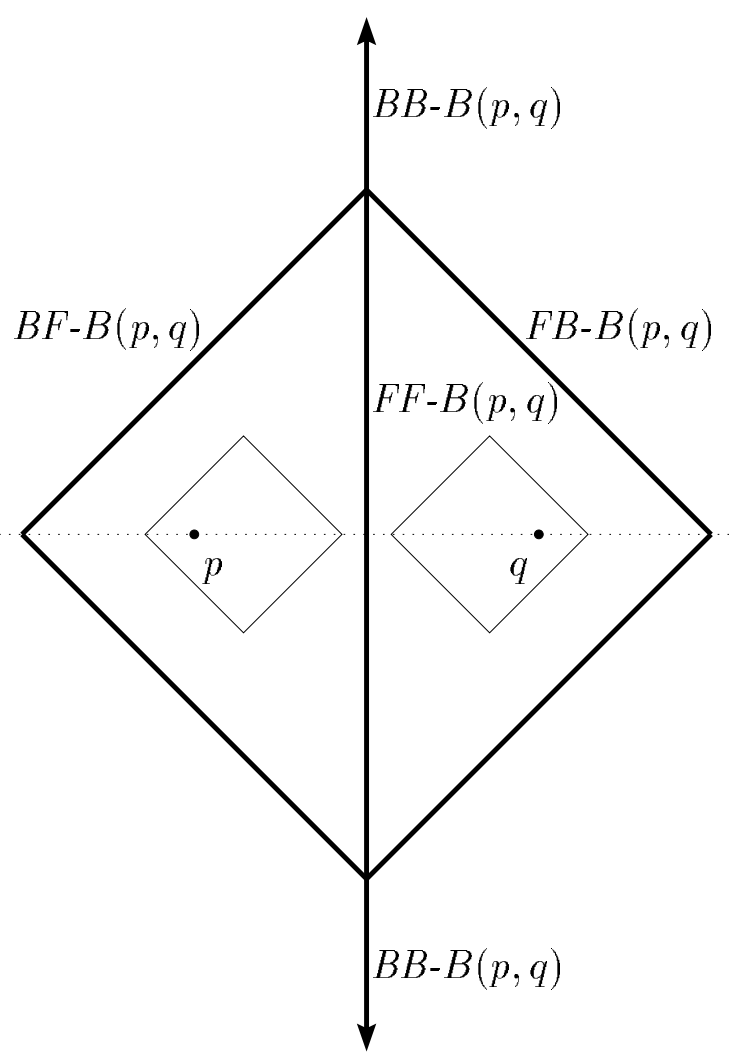

Figure 10: The bisector $B(p, q)$ consists of one connected component.

\section{Polyhedral gauges}

A polyhedral gauge $\gamma_{C}$ is a gauge, whose unit ball $C$ is given by a convex polygon. This class of gauges is important, since a convex set can be approximated with arbitrary $\varepsilon$-accuracy by a convex polyhedron, so the polyhedral gauges are dense in the set of all gauges [12]. In this section, we first develop some special properties of polyhedral gauges, then we show how to compute the corresponding bisector. 


\subsection{Special properties}

Let $C$ be a convex polygon serving as unit ball. The directions from $O$ through the vertices of $C$ are called fundamental directions. Let $v$ and $w$ be two consecutive vertices of $C$. The following lemma shows that $\gamma_{C}$, restricted to the cone between two consecutive fundamental directions, is a linear function, see [13].

Lemma 10 For all points $x=\lambda v+\mu w$, with $\lambda, \mu \geq 0$, in the cone between the two fundamental directions $\overrightarrow{O v}$ and $\overrightarrow{O w}$ we have $\gamma_{C}(x)=\lambda+\mu$.

Now let us consider two convex unit balls $C$ and $D$ with $m$ resp. $n$ vertices. Their fundamental directions from $p$ resp. $q$ partition the plane into at most mn convex cells. A direct consequence of Lemma 10 is the following.

Corollary 11 The bisector $B(p, q)$ is linear in each cell, this means that it is either empty, a point, a line segment, a halfine or the complete cell.

Now let us derive some upper bounds for the complexity of the bisector.

Lemma 12 The bisector $B(p, q)$ contains less than $2(m+n)$ vertices.

Proof. First, we consider FF-B $(p, q)$. From Corollary 11 we know that any vertex of the bisector must lie on the boundary of a cell, i. e. on a fundamental direction from $p$ or $q$. Normally, there is at most one vertex of $F F-B(p, q)$ on each such direction, except for the special case of a whole cell belonging to the bisector. But also in this special case, see Example 5, it is clear that the number of vertices is at most the number of fundamental directions in $F K_{p}$ and $F K_{q}$.

Similar arguments hold for $B F-B(p, q), F B-B(p, q)$, and $B B-B(p, q)$, but these parts are simpler since they do not contain whole cells. Altogether, each fundamental direction contributes at most two vertices to the whole bisector. But this bound is not tight, because the bound for $F F-B(p, q)$ is only tight in the case of two whole cells belonging to the bisector which implies that $B B-B(p, q)$ is not empty and then there is a fundamental direction in a back cone does not contribute a vertex to $B B-B(p, q)$. 
Example 7 Figure 11 shows a bisector consisting of $2(m+n)-8$ vertices which proves that the bound of Lemma 12 is essentially tight. Here, the unit balls are two "D-shaped" convex polygons, all vertices belong to the face cones. $F F-B(p, q)$ contains $m+n-4$ vertices, while $B F-B(p, q)$ and $F B-B(p, q)$ contain $n-2$ resp. $m-2$ vertices, and $B B-B(p, q)$ is empty.

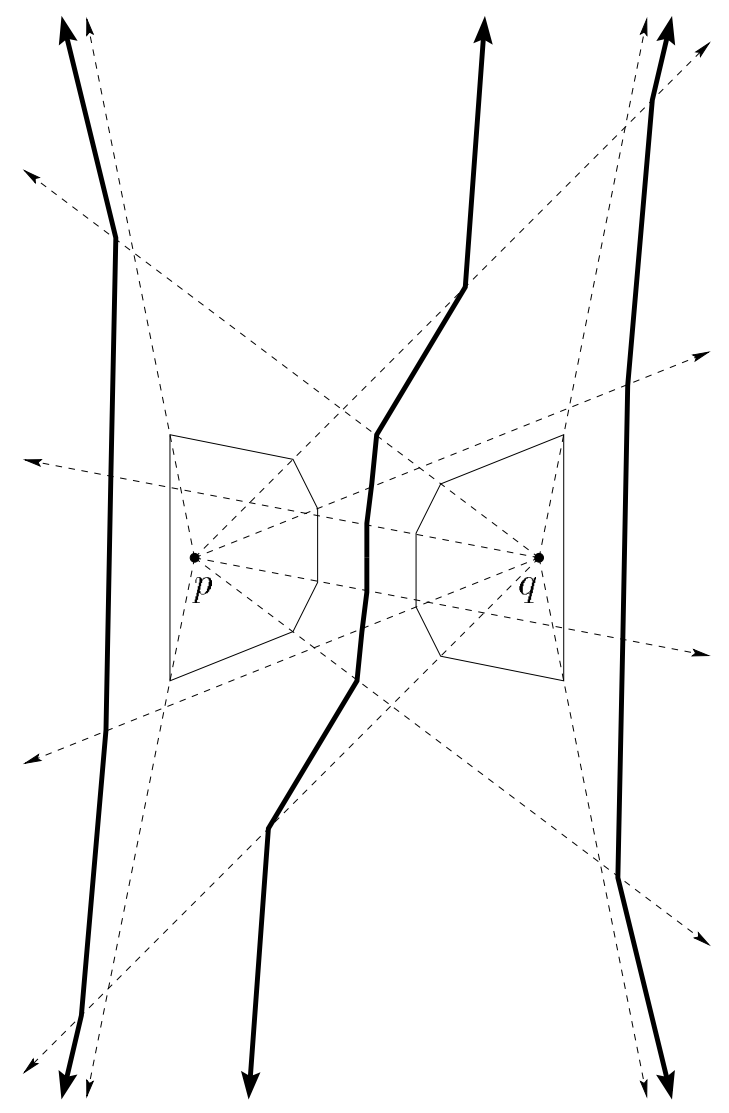

Figure 11: For $m=n=6$ vertices on the unit balls, this bisector consists of $2(m+n)-8=16$ vertices. The example generalizes to any $m, n \geq 3$.

Example 8 Figure 12 shows a situation where the bisector runs through all cells. Of course, such a behaviour is only possible in a situation where the number of cells is less than $2(m+n)$. 


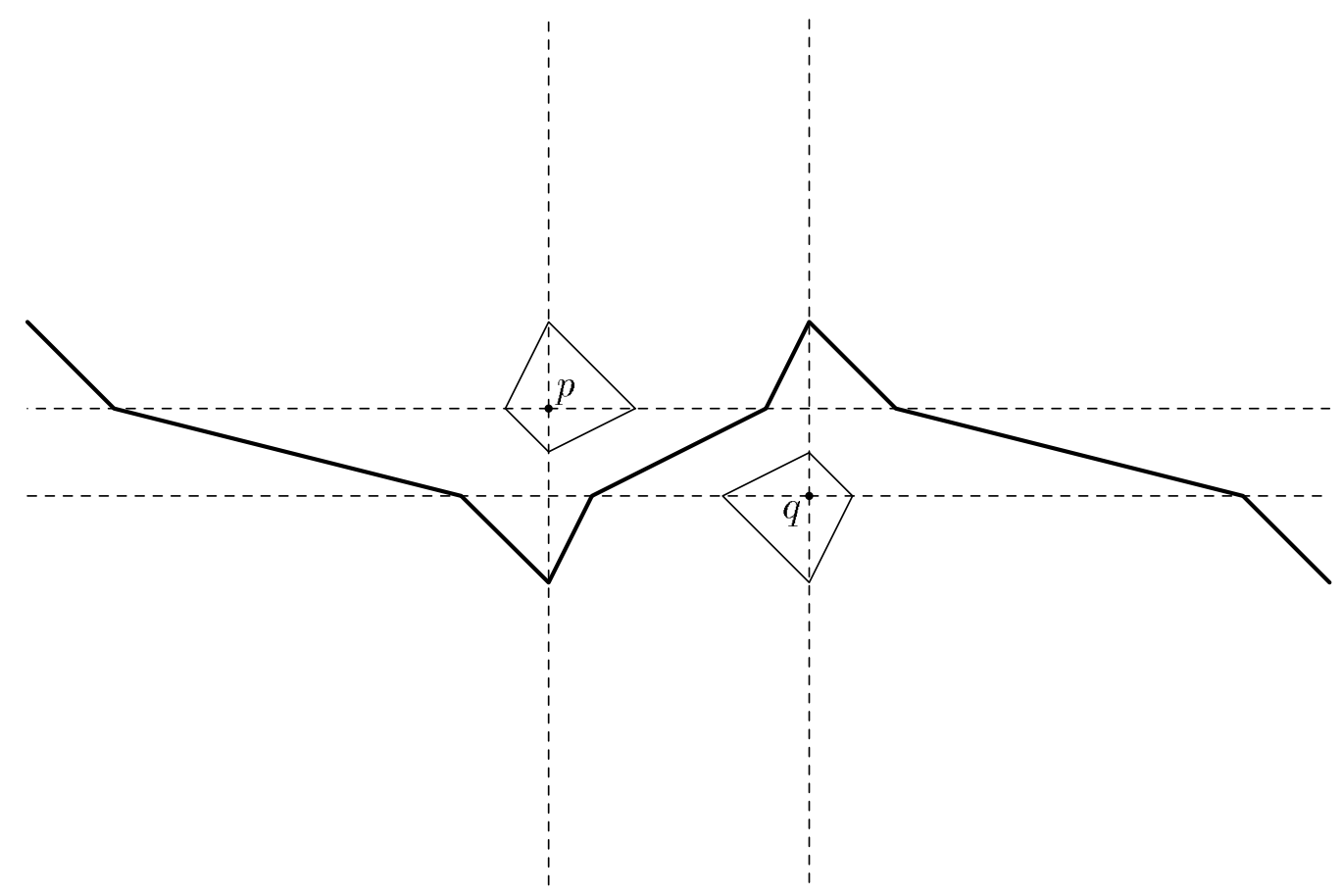

Figure 12: This bisector is homeomorphic to a line and visits all cells.

Lemma $13 B(p, q)$ consists of at most $\min \{m, n\}$ connected components. This bound is tight.

Proof. The boundaries of two convex polygons $C$ and $D$ with $m$ resp. $n$ vertices intersect at most $2 \min (m, n)$ times. Therefore, the set $E$ from Lemma 7 (ii) belonging to $B F-B(p, q)$ and its corresponding counterpart for $F B-B(p, q)$ together have at $\operatorname{most} \min \{m, n\}$ components, but only if all intersections lie in $B K_{p} \cup F K_{q}$ or $F K_{p} \cup B K_{q}$. Now from Corollary 8 follows that the whole bisector consists of at $\operatorname{most} \min \{m, n\}+1$ components.

But $F F-B(p, q)$ is only separated from the rest if two of the intersections lie in $F K_{p} \cup F K_{q}$, due to Lemma 7 (i). This reduces by one the number of possible components of $B F-B(p, q)$ and $F B-B(p, q)$.

The bound is tight, as Example 3 shows. 


\subsection{An algorithm}

As before, we assume $p q$ to be a horizontal line and that point $p$ lies to the left of $q$. In practice, this can be achieved by an appropriate rotation of the coordinate system.

First, let us consider how to compute $F F-B(p, q)$, the part of the bisector in the two face cones. We start by computing the point on $p q$ as described in the proof of Lemma 4 (i), which is guaranteed to in $F F-B(p, q)$. If there is no horizontal fundamental direction then this point is not a real a vertex of the bisector and may be removed at the end.

$$
\begin{aligned}
& x_{0}:=\lambda_{0} p+\left(1-\lambda_{0}\right) q ; \\
& \operatorname{report}\left(x_{0}\right)
\end{aligned}
$$

Reporting a point means that it is a vertex of the bisector which has to be connected to the previously reported vertex, if any.

Using a horizontal sweep line we scan the facing sides of the two unit balls from $O$ upwards until the upper part of $F F-B(p, q)$ is constructed. The lower part is computed in the same manner.

By $\overline{v_{s} v_{e}}$ and $\overline{w_{s} w_{e}}$ we denote the line segments on the boundary of $C$ resp. $D$ that are intersected by the current sweep line. They are initialized in the following way. The successor (succ) of a vertex is the next vertex above on the boundary of the unit ball, i. e. counterclockwise on $\partial C$ and clockwise on $\partial D$.

$v_{e}:=$ the highest vertex of $\partial C \cap F K_{p}$ which lies below or on the line $p q$; $v_{s}:=\operatorname{succ}\left(v_{e}\right)$

$w_{e}:=$ the highest vertex of $\partial D \cap F K_{q}$ which lies below or on the line $p q$; $w_{s}:=\operatorname{succ}\left(w_{e}\right)$;

For advancing the current line segment $\overline{v_{s} v_{e}}$ we use a subroutine which also determines if the $y$-value of the top point $t_{p}$ is already reached. 
procedure advanceV;

$v_{e}:=v_{s}$

$v_{s}:=\operatorname{succ}\left(v_{s}\right) ;\left({ }^{*}\right.$ if there is one in the face cone $\left.{ }^{*}\right)$

if $v_{e}(y)=t_{p}(y)$ then reached_end $:=$ true;

end

There is an analogous procedure advanceW for $\overline{w_{s} w_{e}}$.

Now we describe the main part of the computation. The bisector is constructed one vertex after the other. We make use of Corollary 11, which says that the bisector only changes at fundamental directions. So we step through all of these fundamental directions in the face cones, in the $y$-order of the defining vertices. For each such vertex, we look for its partner foot-point as described in Lemma 1, i. e. we determine the intersection of the horizontal line (horiz) through the vertex and the current segment on the other side. The main loop is performed as long as the maximum $y$-value of one of the unit balls is not reached and the current line segments do not intersect.

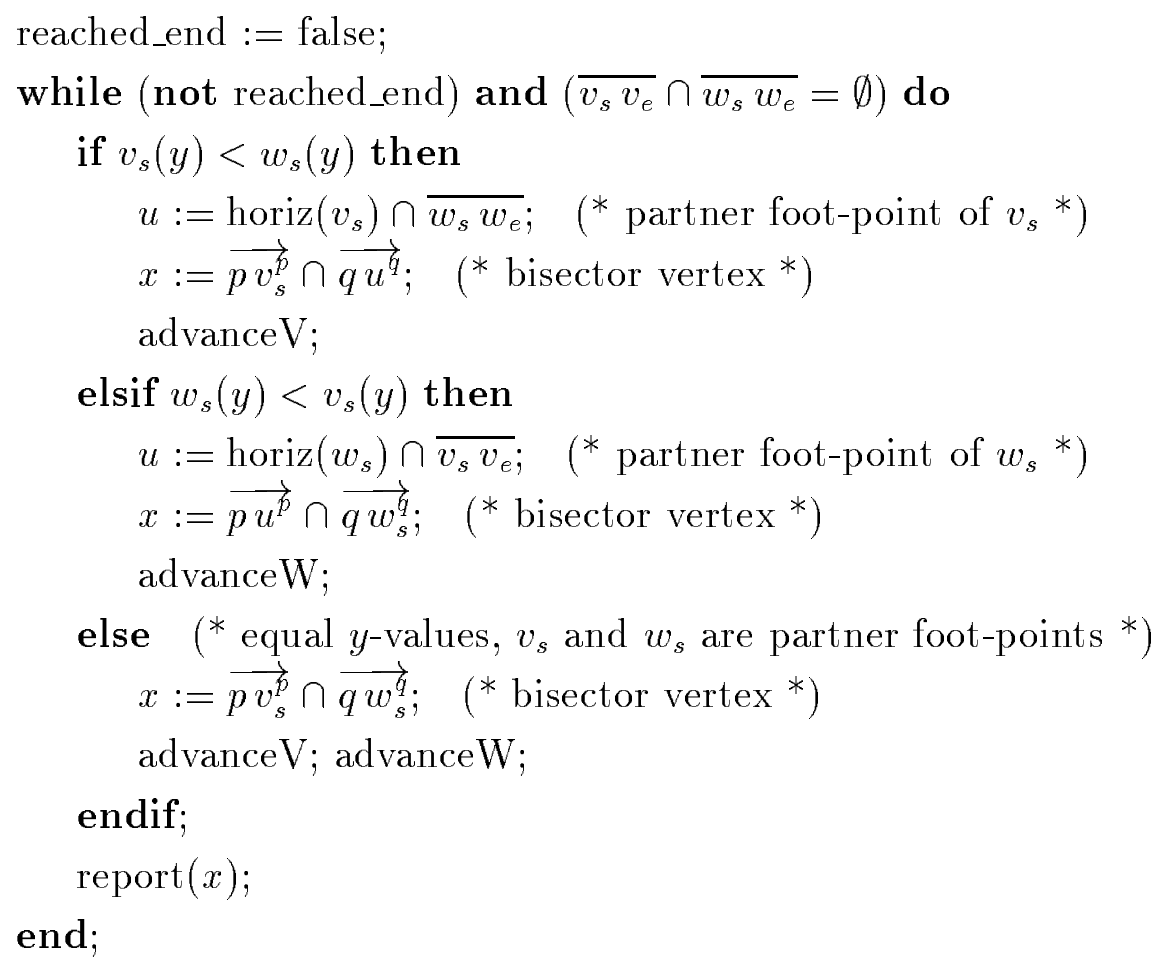


It remains to deal with the final part of $F F-B(p, q)$. Here, some special cases can occur. By cone $(p, \overline{a b})$ we mean the cone consisting of all rays from $p$ through a point of the line segment $\overline{a b}$.

if not reached_end then $\left(* \overline{v_{s} v_{e}}\right.$ and $\overline{w_{s} w_{e}}$ intersect in a point $\left.{ }^{*}\right)$ $s:=\overline{v_{s} v_{e}} \cap \overline{w_{s} w_{e}}$; $\operatorname{report}\left(\overrightarrow{x s^{\underline{k}}}\right) ; \quad\left({ }^{*}\right.$ bisector ends in a ray from $x$ in direction $\left.\overrightarrow{O s} *\right)$

(* otherwise only horizontal boundary pieces can still make trouble: ${ }^{*}$ ) elsif $\overline{v_{s} v_{e}}$ and $\overline{w_{s} w_{e}}$ are collinear (i. e. horizontal) then $\operatorname{report}\left(\operatorname{cone}\left(p, \overline{v_{s}^{p} v_{e}^{p}}\right) \cap \operatorname{cone}\left(q, \overline{w_{s}^{q} w_{e}^{q}}\right)\right)$;

(* this intersection is a whole, bounded or unbounded, cell *) elsif $\overline{v_{s} v_{e}}$ is horizontal then $s:=\operatorname{horiz}\left(v_{s}\right) \cap \overline{w_{s} w_{e}}$ report $\left(\operatorname{cone}\left(p, \overrightarrow{v_{s}^{p} v_{e}^{p}}\right) \cap \overrightarrow{q s^{q}}\right)$;

elsif $\overline{w_{s} w_{e}}$ is horizontal then

$s:=\operatorname{horiz}\left(w_{s}\right) \cap \overline{v_{s} v_{e}}$ $\operatorname{report}\left(\operatorname{cone}\left(q, \overline{w_{s}^{p} w_{e}^{p}}\right) \cap \overrightarrow{p s^{p}}\right) ;$

endif;

In the last two cases, the reported piece of the bisector is a line segment or a ray.

For constructing the other parts of the bisector we proceed in a very similar way, only the differences are briefly mentioned here.

The construction of the two parts of $B B-B(p, q)$ can take place directly after the construction of the upper resp. lower parts of $F F-B(p, q)$. For the upper part, we start with a sweep line at the last, i.e. maximum, $y$-value. The upper part of $B B-B(p, q)$ exists iff this line intersects the back side of $C$ to the right of the back side of $D$. We scan downwards the two back sides of the unit balls as in the while-loop above until they intersect.

For $B F-B(p, q)$ and $F B-B(p, q)$ we start again at the $y$-value where the computation of $F F-B(p, q)$ has come to an end. For $B F-B(p, q)$, we scan along 
the back side of $C$ and the face side of $D$. Bisector pieces are generated while the face side of $D$ lies to the left of the back side of $C$, i. e. $\gamma_{q}$ is stronger. The computation does not end at an intersection of the two chains, as it was the case for $F F-B(p, q)$, but each intersection produces a ray of the bisector instead. As long as $\gamma_{p}$ is stronger, no bisector pieces arise, but we search for the next intersection. This is continued until the minimum $y$-value is reached.

Theorem 14 For two points, $p$ and $q$, in the plane and two associated polyhedral gauges $\gamma_{p}$ and $\gamma_{q}$ with $m$ resp. $n$ fundamental directions, our algorithm computes the bisector $B(p, q)$ in optimal time $O(m+n)$.

Proof. Essentially, we perform four scans through the vertices of the unit balls, one for each of the four parts of the bisector. Therefore, the number of steps in the algorithm is bounded by a constant times the number of all vertices, provided that the vertices of the two polygons are given in, say clockwise, order.

It is clear that $\Omega(m+n)$ time is sometimes necessary, see Examples 3 and 7. But even if the number of vertices of the bisector is small, the scans for constructing $B F-B(p, q)$ and $F B-B(p, q)$ may pass through a very large number of vertices. This is unavoidable. For our problem there is no outputsensitive algorithm, i. e. one whose running time only depends on the size of the output. To see this, we slightly modify the two $n$-gons in Example 3 such that only a few of the intersections really happen. Then nearly every edge of the two polygons must be inspected at least once, while the bisector is quite simple.

\section{Conclusions}

We have presented a first investigation about bisectors of two different con-

vex distance functions (gauges). Precise characterizations were given about 
which parts of the two unit balls contribute to the bisector, how many connected components it consists of, and how the different parts of the bisector look like. We have seen that the intersection of the unit balls essentially determines its behaviour. Examples were given for interesting situations like many disconnected pieces, 2-dimensional areas contained in the bisector, and "weak" bisector pieces which do not separate different regions.

For polyhedral gauges, the complexity of the bisector can be bounded in terms of the number of vertices of the unit balls. An optimal linear time algorithm has been presented for computing such a bisector.

To continue this research about different gauges the next steps should concern the Voronoi diagram of many sites where each site is associated its own gauge. Taking into account the sometimes strange characteristics of the bisector of two sites, which we have observed in this paper, it seems clear that the Voronoi diagram will be pretty complicated.

\section{References}

[1] F. Aurenhammer and H. Edelsbrunner. An optimal algorithm for constructing the weighted Voronoi diagram in the plane. Pattern Recogn., $17: 251-257,1984$.

[2] F. Aurenhammer and R. Klein. Voronoi diagrams. In J.-R. Sack and J. Urrutia, editors, Handbook of Computational Geometry. Elsevier Science Publishers B.V. North-Holland, Amsterdam, 1998.

[3] L. P. Chew and R. L. Drysdale, III. Voronoi diagrams based on convex distance functions. In Proc. 1st Annu. ACM Sympos. Comput. Geom., pages 235-244, 1985.

[4] A. G. Corbalan, M. Mazon, and T. Recio. Geometry of bisectors for strictly convex distances. Internat. J. Comput. Geom. Appl., 6:45-58, 1996. 
[5] H. Edelsbrunner and R. Seidel. Voronoi diagrams and arrangements. Discrete Comput. Geom., 1:25-44, 1986.

[6] H. W. Hamacher. Mathematische Lösungsverfahren für planare Standortprobleme. Verlag Vieweg, Wiesbaden, 1995.

[7] C. Icking, R. Klein, N.-M. Lê, and L. Ma. Convex distance functions in 3-space are different. Fundam. Inform., 22:331-352, 1995.

[8] P. Kelly and M. Weiss. Geometry and Convexity: A Study in Mathematical Methods. Wiley, New York, NY, 1979.

[9] S. Nickel, J. Puerto, A. Rodriguez-Chia, and A. Weißler. General continuous multicriteria location problems. Report in Wirtschaftsmathematik 28, University of Kaiserslautern, Department of Mathematics, 1997.

[10] A. Okabe and A. Suzuki. Locational optimization problems solved through Voronoi diagrams. European Journal of Operational Research, pages $445-456,1997$.

[11] A. Rodriguez-Chia, S. Nickel, and J. Puerto. A flexible approach to location problems. Report in Wirtschaftsmathematik 26, University of Kaiserslautern, Department of Mathematics, 1997.

[12] F. A. Valentine. Convex Sets. McGraw-Hill, New York, 1964.

[13] J. E. Ward and R. E. Wendell. Using block norms for location modeling. Oper. Res., 33:1074-1090, 1985.

[14] P. Widmayer, Y. F. Wu, and C. K. Wong. Distance problems in computational geometry with fixed orientations. In Proc. 1st Annu. ACM Sympos. Comput. Geom., pages 186-195, 1985. 